\title{
Abnormal Functional Connectivity Density in Patients with Dysthyroid Optic Neuropathy
}

\author{
Yunhai Tu ${ }^{a}$ Pingping Huang ${ }^{b}$ Chuanwan Mao ${ }^{c}$ Xiaozheng Liu ${ }^{c}$ Jianlu Gao ${ }^{a}$ \\ aLiaocheng People's Hospital, Cheeloo College of Medicine Shandong University, Liaocheng, China; ${ }^{\text {bThe Eye }}$ \\ Hospital, Wenzhou Medical University, Wenzhou, China; 'Department of Radiology, Yuying Children's Hospital, \\ The Second Affiliated Hospital of Wenzhou Medical University, Wenzhou, China
}

\section{Keywords}

Functional connectivity density · Dysthyroid optic neuropathy - Thyroid-associated ophthalmology

\begin{abstract}
Objective: Functional connectivity density (FCD) mapping was used to investigate abnormalities and factors related to brain functional connectivity in cortical regions of patients with dysthyroid optic neuropathy (DON) and to analyze the pathogenesis of DON further. Methods: Patients diagnosed with thyroid-associated opthalmology (TAO) in the Eye Hospital were enrolled. All patients underwent comprehensive eye examinations and best-corrected visual acuity, visual field (VF) test. MRI data collection and analysis were completed in the 2nd Affiliated Hospital of Wenzhou Medical University. The patients were divided into 2 groups: the DON group, with an average VF, mean deviation (MD) of both eyes $<-5 \mathrm{~dB}$, and the non-DON group (nDON group), with an average VF MD of both eyes $\geq-2 \mathrm{~dB}$. Results: $A$ total of 30 TAO patients (14 men, 16 women) with complete data who met the experimental requirements were enrolled. The average age was 48.79 (40-57) years. There were 16 patients in the DON group and 14 patients in the nDON group. No significant differences in age, gender, education level, and the maximum horizontal diameter of either medial rectus mus-
\end{abstract}

cle were found between the 2 groups. The difference of brain FCD between the 2 groups showed significant abnormal connectivity in the right orbital gyri of the frontal lobe (Frontal_Inf_Orb_R) and the left precuneus in the DON group compared with the nDON group. As demonstrated by decreased FCD values in the right inferior frontal gyrus/orbital part, the relevant brain regions were the left middle temporal gyrus, left precuneus, left middle frontal gyrus, right postcentral gyrus, and brain gyri (excluding the supramarginal gyrus and angular gyrus) below the left parietal bone. The FCD associated with the left precuneus was increased, and the relevant brain areas were the left middle temporal gyrus, right cuneus, superior occipital gyrus, and right fusiform gyrus. A significant correlation was identified between the MD of the binocular VF and brain FCD. Conclusion: The abnormal FCD in the cortex of DON patients suggests that a central nervous system mechanism may be related to the pathogenesis of the DON.

(c) 2020 The Author(s)

Published by S. Karger AG, Basel

\section{Introduction}

Dysthyroid optic neuropathy (DON) is a severe complication of thyroid-associated opthalmology (TAO) [1]. The early clinical futures include abnormal color vision karger@karger.com www.karger.com/ore

Karger $\stackrel{\text { ' }}{=}$

GOPEN ACCESS
(C) 2020 The Author(s)

Published by S. Karger AG, Basel

This is an Open Access article licensed under the Creative Commons Attribution-NonCommercial-4.0 International License (CC BY-NC) (http://www.karger.com/Services/OpenAccessLicense), applicable to the online version of the article only. Usage and distribution for commercial purposes requires written permission.
Correspondence to:

Jianlu Gao,drgaojianlu@163.com 
and contrast sensitivity, and the late manifestations are irreversible loss of vision or even blindness. It can be seen that the late stage of the DON will severely impair visual function, so it is critical to understand the pathogenic mechanism and symptomatic treatment of the DON.

The current mainstream point is that the DON results from the compression of the optic nerves caused by orbital apex crowding. However, we observed that not all patients with DON presented with orbital apex crowding. In previous studies, some researchers have studied indicators that reflect orbital apex crowding in patients with DON, such as extraocular muscle parameters, the degree of dilation of the superior ophthalmic veins, and the degree of fat herniation through the superior orbital fissure. Although there are significant differences between DON and non-DON (nDON) patients, the ranges of these indicators overlap significantly between the 2 groups. When reviewing the literature, we noticed that different studies are not consistent or even contradictory [2-5]. According to the orbital apex crowding theory of orbital apex crowding, orbital decompression is an effective treatment for DON, but persistent vision loss has been reported in patients with DON who underwent orbital decompression $[6,7]$. Therefore, we hypothesize that in addition to crowding of the orbital apex, other pathogenesis mechanisms may promote the development of the DON.

Thyroid hormones are essential for maintaining the structures and functions of adult visual pathways. Clinical epidemiology has shown that the incidence of openangle glaucoma in patients with thyroid-associated diseases is higher than that of a healthy population [8] and that abnormal thyroid function is a high-risk factor for age-related macular degeneration $[9,10]$. Clinical observation also shows that patients with hypothyroidism have abnormal color contrast sensitivity [11], abnormal VEP $[12,13]$, and VF defects [14]. These clinical manifestations are very similar to DON. Related basic research further revealed that thyroid hormone abnormalities could cause abnormal opsin expression in retinal cone cells [15], abnormal proliferation and differentiation of optic nerve oligodendrocytes, and even demyelination [1618]. Clinical observation found that thyroid dysfunction is closely related to the development and progress of TAO. Furthermore, some scholars believe that thyroid dysfunction is a potential risk factor of DON [1]. Therefore, we speculate that the dysfunction of thyroid hormones may be involved in the pathogenesis of the DON.

A resting-state functional magnetic resonance imaging (rs-fMRI) study showed that adult patients with thyroid dysfunction had brain abnormalities, including gray matter density, cerebral blood flow, and brain connectivity [19-27]. If dysfunction of thyroid hormone is involved in the pathogenesis of the DON, then patients with DON will also show brain abnormalities similar to those patients with dysfunction of thyroid hormone. However, as far as we know, there is no study on the rs-fMRI of the DON (Retrieval strategy: PubMed database, retrieval method-dysthyroid optic neuropathy [af]) and fMRI[af])

Functional connectivity density (FCD) mapping is a graph-based measurement of network organization that reflects the number of instantaneous functional connections between a region and the rest of the brain within the entire connectivity matrix of the brain. Thus, it can assess how much a node influences the entire brain and integrates information across functionally isolated brain regions [28]. Therefore, this article intends to use the FCD method of rs-fMRI for the brain to explore whether there is abnormal functional brain connectivity in patients with DON and its correlation with clinical features of DON.

\section{Experimental Methods}

Patient enrollment: patients diagnosed with TAO in the Eye Hospital, Wenzhou Medical University, from January 2018 to December 2019 were enrolled (referring to the internationally recognized diagnostic criteria proposed by Bartley and Gorman [29]). The study protocol was approved by the Ethics Committee Board of the Eye Hospital of Wenzhou Medical University and adhered to the Declaration of Helsinki's tenets. Consent from every patient was obtained before being included in the study; DON was diagnosed in TAO patients if they had at least 2 of the following signs related to optic neuropathy: vision loss, VF defects, relative afferent pupillary defects (RAPD) (+), and optic disc edema. All patients underwent best-corrected visual acuity testing, and the results were expressed as decimals. A Humphrey VF analyzer was used for measuring the VF, and the mean deviation (MD, refers to the average amount of increase or decrease in the sensitivity of VF detection compared with the normal value) was recorded. Orbital MRI was performed to determine the parameters of rectus muscles (Barrett L. 1988) and the maximum diameters of both medial rectus muscles (Weis et al. [5]).

\section{Inclusion Criteria}

Patients who met the following criteria were included: (1) age 40-60 years; (2) right-handedness; (3) schooling $\geq 5$ years; (4) good physical condition with the ability to walk (with or without assistance) and complete the audiovisual test; (5) willingness to voluntarily cooperate with the research protocol and sign an informed consent form after fully understanding the safety of the research project.

\section{Exclusion Criteria}

Patients who met one of the following criteria were excluded: (1) eye diseases that may affect visual function, such as corneal ul- 
Table 1. General data of the patients

\begin{tabular}{llll}
\hline & DON group & nDON group & $p$ value \\
\hline Gender (M/F) & $16(10 / 6)$ & $14(9 / 5)$ & 1.000 \\
Years of education & $5.93 \pm 2.48^{*}$ & $8.50 \pm 4.84^{*}$ & 0.084 \\
MD, VF OD & $-12.71 \pm 11.11^{*}$ & $-1.58 \pm 1.81^{*}$ & 0.000 \\
MD, VF OS & $-11.46 \pm 8.12^{*}$ & $-1.32 \pm 1.42^{*}$ & 0.000 \\
Degree of exophthalmos, OD & $20.23 \pm 3.05^{*}$ & $17.85 \pm 1.99^{*}$ & 0.031 \\
Degree of exophthalmos, OS & $21.58 \pm 2.88^{*}$ & $18.25 \pm 3.38^{*}$ & 0.014 \\
Maximum diameter of the medial rectus, OD & $6.40 \mathrm{~mm}$ & $5.76 \mathrm{~mm}$ & 0.15 \\
Maximum diameter of the medial rectus, OS & $6.23 \mathrm{~mm}$ & $5.58 \mathrm{~mm}$ & 0.16 \\
\hline
\end{tabular}

$M$, male; $F$,female; $V F$, visual field; $M D$, mean deviation; $D O N$, dysthyroid optic neuropathy. * Data are expressed as the mean \pm standard deviation.

cers, cataracts, retinal vascular diseases, retinal detachment, and other optic nerve diseases; (2) history of surgery due to other eye diseases, orbital decompression, and eye radiation therapy; (3) acute TAO or lagophthalmos; (4) history of diabetes or hypertension; (5) history of brain tumor, brain trauma, or cerebrovascular disease; (6) history of nervous system disease or mental disease or family history of these diseases; (7) poorly controlled diabetes or severe major systemic diseases such as heart, liver, or kidney insufficiency.

\section{Grouping}

According to the VF MD, the patients were divided into 2 groups: the DON group, in which the average VF MD of both eyes was $<-5 \mathrm{~dB}$, and the nDON group, in which the average VF MD of both the eyes was $\geq-2 \mathrm{~dB}$.

\section{MRI Data Acquisition}

The Signa HDxt 3.0 T MRI scanner (G.E. Corp., USA) with eight-channel head coils and spine-specific imaging coils was used to acquire MRI data of the head and spinal cord, respectively. During the scanning, the subject was at rest in the supine position and awake with the eyes closed. The scanning sequence was (1) conventional cranial MRI sequences (slice thickness, $5.0 \mathrm{~mm}$; the number of slices, 20): axial T1-weighted imaging (T1WI), T2weighted imaging (T2WI), and T2WI-fluid-attenuated inversion recovery (FLAIR); (2) resting-state blood oxygenation level-dependent (BOLD) MRI sequence: gradient-echo echo-planar imaging sequence (repetition time (T.R.), 2,000 ms; echo time (T.E.), 30 ms; flip angle (F.A.), $90^{\circ}$; slice thickness, $3 \mathrm{~mm}$; interslice gap, 1 $\mathrm{mm}$; the number of slices, 33; matrix, $64 \times 64$; the field of view (FOV), $200 \times 200 \mathrm{~mm}$, and 240-time points); (3) three-dimensional whole-brain high-resolution T1WI (T.R., $8.3 \mathrm{~ms}$; T.E., $3.3 \mathrm{~ms}$; F.A., $12^{\circ}$; slice thickness, $1 \mathrm{~mm}$, interslice gap, $0 \mathrm{~mm}$, number of slices, 156; matrix, $256 \times 256$; FOV, $240 \times 240 \mathrm{~mm}$ ); (4) routine spinal MRI sequence: sagittal T1WI, T2WI, short-tau inversion recovery (STIR), and axis T2WI.

\section{MRI Data Processing}

Resting-state BOLD data were processed by using DPARSFA (v2.3, http://www.restfmri.net) software as follows: (1) We removed the data from the first ten time points (to exclude the effects of environmental interference on subjects) and performed slice timing and alignment (excluding $3 \mathrm{DON}$ patients with head mo- tions, namely, horizontal motion $>2 \mathrm{~mm}$ and/or rotation $>2^{\circ}$ ). (2) We performed spatial normalization by converting the data into the Montreal Neurological Institute space and resampling the data to $3 \times 3 \times 3 \mathrm{~mm}$ voxels. (3) A Gaussian abnormal FCD smooth kernel smoothed normalized data with a full-width at half-maximum value of $6 \mathrm{~mm}$. (4) We used a bandpass filter of $0.01-0.08 \mathrm{~Hz}$ to remove high-frequency noise. (5) We used multiple linear regression analysis to remove covariates, including 6 head motion parameters, cerebrospinal fluid templates, and white matter templates.

Pearson correlation analysis was used to calculate the FCD between a given voxel and other voxels within a gray matter mask. The FCD maps were obtained by setting a threshold correlation coefficient $r>0.25[30,31]$. We used only the positive binarized FCD values for statistical analysis. DPARSFA software was used to perform F.C. analysis on the seed points to study the relevant brain areas that caused FCD differences. The coordinates of brain areas with intergroup differences which were obtained from FCD values were selected as seeds for further F.C. analyses were extracted. Seeds were constructed by drawing a 6- $\mathrm{mm}$ radius sphere around the center voxels of these regions, and the time series for each seed was extracted from the preprocessed data. Time series were averaged across all voxels within each seed's sphere. Pearson's correlation analyses were estimated between the seeds and the remaining voxels. REST Software was used to view the images and extract the brain areas with intergroup differences as a mask for later comparison.

\section{Statistical Analysis}

REST (v1.9, http://www.restfmri.net) was used to perform twosample $t$-tests on the 2 groups, and changes in FCD values in each brain area between the groups were analyzed. AlphaSim was used to correct for multiple comparisons. AlphaSim is part of a standard neuroimaging toolbox, AFNI (http://afni.nimh.nih.gov/), which uses Monte Carlo permutation simulations to estimate the overall significant level achieved. The corrected $p$-value threshold was set at 0.05 ( $p<0.005$ for a single voxel; a cluster was defined as $>30$ voxels). REST was used to extract FCD values of brain areas with intergroup differences. SPSS 25.0 software was used to calculate the Pearson correlation coefficients between these FCD values and the MD values of the VF - the optical coherence tomography (OCT) results. $p<0.05$ with false discovery rate correction was considered statistically significant. 
Table 2. The difference in VF defect between the DON group and the nDON group

\begin{tabular}{llll}
\hline OCT metrics & DON group & nDON group & $p$ value \\
\hline Nasal OD & $71.60 \pm 14.86$ & $73.90 \pm 8.41$ & 0.675 \\
$\quad$ OS & $76.90 \pm 17.11$ & $78.50 \pm 10.08$ & 0.754 \\
Temporal OD & $80.00 \pm 20.97$ & $88.60 \pm 11.41$ & 0.270 \\
$\quad$ OS & $73.60 \pm 18.79$ & $84.50 \pm 11.02$ & 0.131 \\
Superior OD & $142.90 \pm 43.96$ & $132.40 \pm 22.69$ & 0.551 \\
$\quad$ OS & $148.10 \pm 43.79$ & $150.20 \pm 15.77$ & 0.888 \\
Inferior OD & $133.20 \pm 34.73$ & $137.70 \pm 15.29$ & 0.712 \\
$\quad$ OS & $127.80 \pm 30.79$ & $141.90 \pm 13.84$ & 0.203 \\
\hline
\end{tabular}

VF, visual filed; OCT, optical coherence tomography.

\section{Experimental Results}

\section{The Screening of TAO Patients}

A total of 30 TAO patients ( 14 men, 16 women) with complete data who met the inclusion criteria were enrolled from January 2018 to December 2019. There were 16 patients in the DON group and 14 patients in the nDON group. There was no significant difference in age, gender, year of education, and the maximum diameter of either medial rectus muscle between the 2 groups (as shown in Table 1 for details). The mean MD values of the right and left eyes were -8.37 and -7.07 , respectively, and there was no significant difference between the left and right eyes.

\section{Relationship between the VF and OCT Results}

The difference in VF defects between the DON group and the nDON group was statistically significant. Although the thicknesses of the nasal, temporal, superior, inferior, central VF, and the average nerve fiber layer in the Don group were smaller than those in the $\mathrm{nDON}$ group, there was no statistically significant difference in OCT between the 2 groups (as shown in Table 2).

\section{Analysis of Differences in Brain Connectivity between the Two Groups}

The analysis of the difference in brain connectivity between the 2 groups showed significantly abnormal connectivity in the right orbital gyrus of the frontal lobe and the left precuneus in the DON group compared with the nDON group (shown in Fig. 1). The main manifestation was the decline in the brain connectivity of the right orbital part of the inferior frontal gyrus, and the relevant brain areas were the left middle temporal gyrus, the left precuneus, the left middle frontal gyrus, and the postcen- tral gyrus. There was an increase in connectivity associated with the left precuneus, which was related to the left middle temporal gyrus, the right cuneus, and the right fusiform gyrus (shown in Table 3).

Correlation between VF, OCT Results, and Brain FCD

There was no correlation between OCT results and brain connectivity. There were significant correlations between the binocular VF MD and the intensity of brain connectivity. The MD of the right VF was negatively correlated with the FCD values in the right orbital gyri of the frontal lobe (Frontal_Inf_Orb_R) $(R=0.490 p=0.008$, as shown in Fig. 2a) and positively correlated with the FCD values in the left precuneus $(R=-0.489 p=0.006$, as shown in Fig. 2b). The MD of the left VF was negatively correlated $(R=-0.481 p=0.011$, as shown in Fig. $2 \mathrm{~d})$ and nonsignificantly positively correlated with FCD values in the Frontal_Inf_Orb_R $(R=0.273 p=0.168$, as shown in Fig. 2c).

\section{The Relationship between Thyroid Hormones and \\ Brain Connectivity Data}

The thyroid hormone levels of all patients were not significantly correlated with the FCD values of different brain areas.

\section{Discussion}

This study focused on the brain connectivity of patients with DON and found that the brain functional connectivity associated with the left precuneus was higher in the DON patients than in the $\mathrm{nDON}$ patients. Moreover, the relevant brain areas were the left middle temporal gyrus, the right cuneus, the right fusiform gyrus, and the left superior occipital gyrus. The FCD decreased in the orbital part of the inferior frontal gyrus. The relevant brain areas were the left middle temporal gyrus, the left precuneus, the left middle frontal gyrus, the postcentral gyrus, and the left inferior parietal cortex. The change in brain connectivity was correlated with the VF MD.

The higher level cortical pathways of human vision include ventral and dorsal pathways [32-34]. Functionally, the dorsal pathways are mainly related to the perception of movements and the spatial positions of objects and participate in the control of eye movements. The ventral pathways are mainly related to the perception of the shapes and colors of objects. Anatomically, the dorsal and ventral pathways originate from the striated cortex of the 


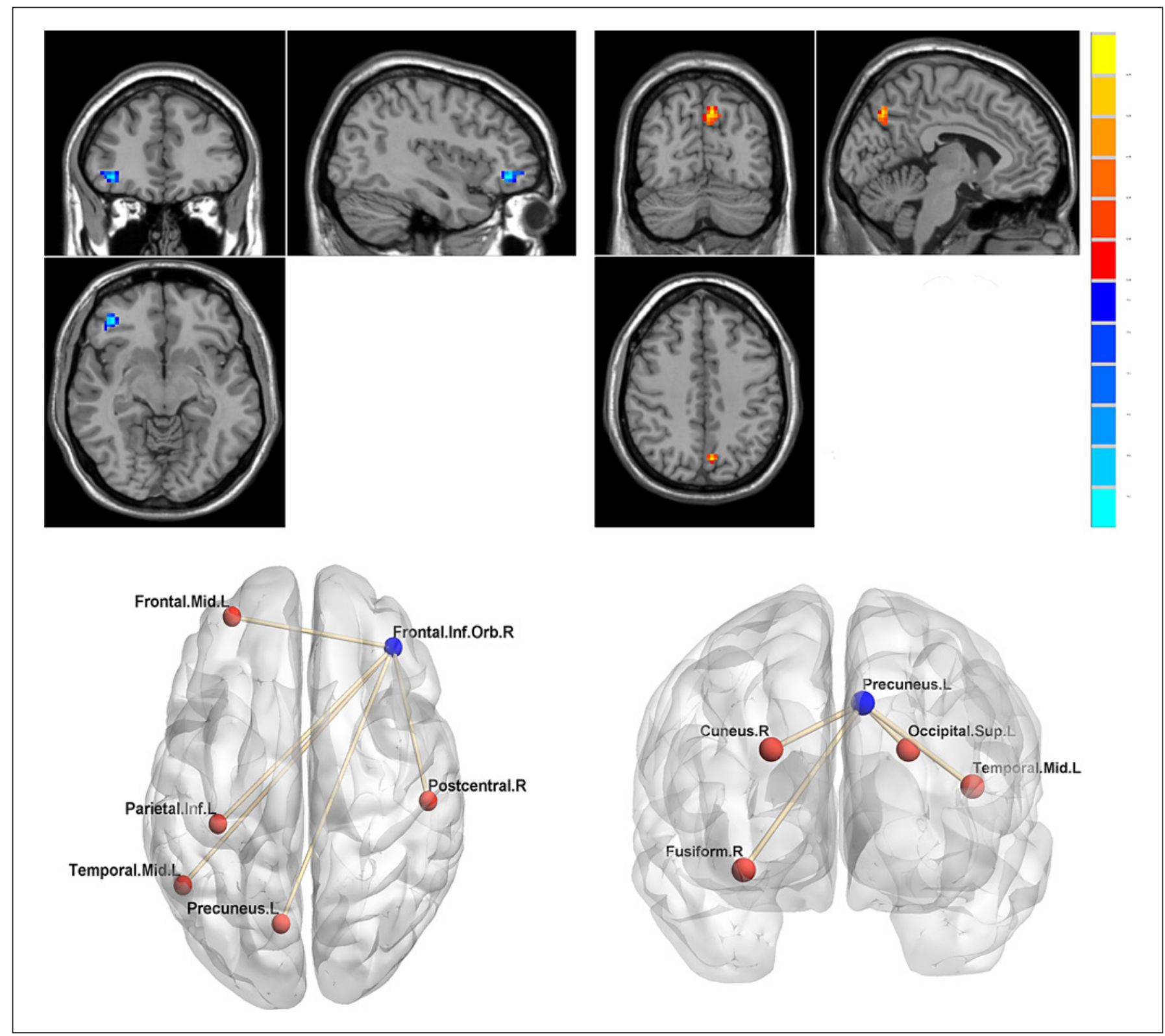

Fig. 1. Three-dimensional MR images showing brain areas with abnormal brain connectivity and the relevant brain regions. Top image: abnormal brain regions after FCD analysis between DON and $\mathrm{nDON}$. Statistical t map is overlapped on the standard T1 template in MNI space. Colorbar corresponding to the $t$ values. Bot-

occipital lobe, and the dorsal pathways extend through the occipital parietal cortex to the inferior parietal lobule and ultimately extend to the dorsal lateral prefrontal lobe. The ventral pathway reaches the anterior temporal cortex through the occipitotemporal cortex and finally extends to the ventral frontal cortex. The dorsal pathway includes 3 pathways through the parietal lobe [32]. The parietal

A New Pathogenesis Hypothesis of DON tom image: the relevant brain regions induced the alternation of FCD between DON and nDON. FCD, functional connectivity density; DON, dysthyroid optic neuropathy; MNI, Montreal Neurological Institute.

lobe-premotor cortex pathway is involved in the control and activation of eye movement and the top-down signal processing of visual information. The parietal-temporal gyrus pathway provides spatial information to the medial temporal lobe and participates in visual-spatial information processing through the posterior cingulate cortex (PCC) and retrosplenial cortex. 
Table 3. Brain areas with intergroup differences in brain connectivity and the relevant brain areas

\begin{tabular}{|c|c|c|c|c|c|c|c|}
\hline \multirow[t]{2}{*}{ Seed regions } & \multirow[t]{2}{*}{ Brain area } & \multirow[t]{2}{*}{ Voxels } & \multirow[t]{2}{*}{$\mathrm{BA}$} & \multicolumn{3}{|c|}{ MNI coordinate } & \multirow[t]{2}{*}{$T$ value } \\
\hline & & & & $x$ & $\mathrm{Y}$ & Z & \\
\hline \multirow[t]{4}{*}{ Left precuneus } & Left middle temporal gyrus & 97 & & -42 & -66 & 15 & 5.9344 \\
\hline & Right cuneus & 153 & & 24 & -66 & 27 & 4.7112 \\
\hline & Right fusiform gyrus & 52 & & 33 & -36 & -12 & 4.4417 \\
\hline & Left superior occipital gyrus & 96 & & -21 & -72 & 27 & 5.2326 \\
\hline \multirow{5}{*}{$\begin{array}{l}\text { Right inferior frontal } \\
\text { gyrus/orbit part }\end{array}$} & Left middle temporal gyrus & 51 & & -51 & 54 & 3 & -5.9439 \\
\hline & Left precuneus & 79 & & -9 & -69 & 51 & -5.8236 \\
\hline & Left middle frontal gyrus & 54 & & -30 & 51 & 15 & -6.3985 \\
\hline & Right postcentral gyrus & 86 & & 54 & -21 & 39 & -5.4101 \\
\hline & Left inferior parietal gyrus & 113 & & -36 & -30 & 33 & -5.2610 \\
\hline
\end{tabular}

BA, Brodmann area; MNI, Montreal Neurological Institute.
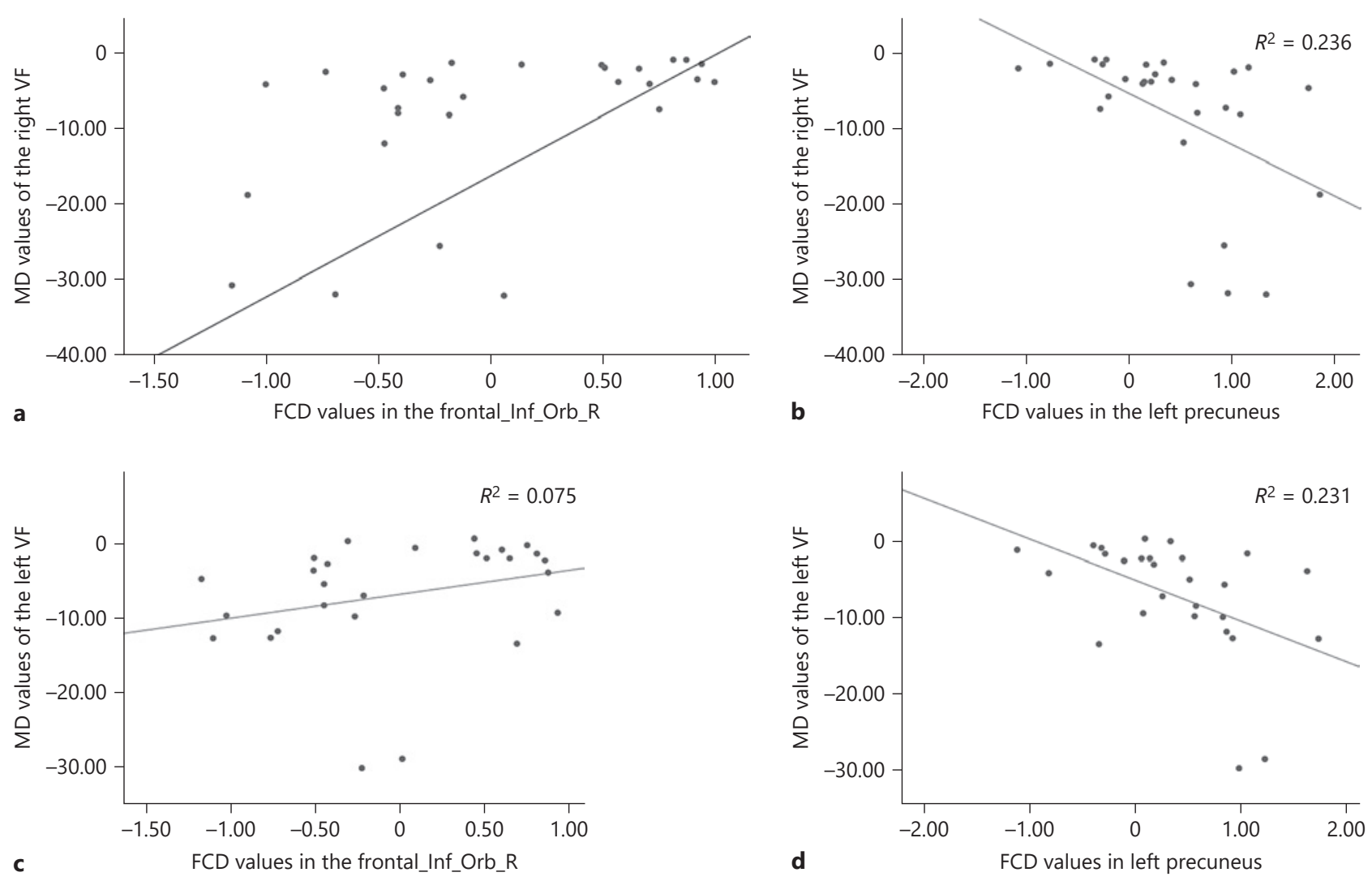

Fig. 2. Scatter plots of the relationships between the MDs of the left and right VF and FCD values. $\mathbf{a}$ is the scatter plot of the MD of the right VF and FCD values in the Frontal_Inf_Orb_R. $\mathbf{b}$ is the scatter plot of the MD of the right VF and FCD values in the left precu- neus. $\mathbf{c}$ is the scatter plot of the MD of the left VF and FCD values in the Frontal_Inf_Orb_R.d is the scatter plot of the MD of the left VF and FCD values in the left precuneus. FCD, functional connectivity density; VF visual field; MD, mean deviation. 
Central visual information processing, in addition to the down-top processing described above, that is, the process from the retina-primary visual cortex to the higher level visual cortex, also includes endogenous top-down visual information processing [35-37]. The prefrontal lobe is essential in advanced brain function. It includes round-trip fiber connections to all sensory areas and participates in the processing and integration of sensory information, especially in top-down control. Bar et al. [38] found that the orbitofrontal gyrus is the origin of the topdown control in the process of visual cognition. This study showed that the FCD values in the right orbitofrontal gyrus were lower in the $\mathrm{DON}$ group than in the $\mathrm{nDON}$ group, which are directly involved in the visual-spatial network of top-down visual information processing.

The precuneus is the brain area with the highest resting metabolic rate. Its functions can be divided into visual-spatial imagery, episodic memory extraction, self-processing, and consciousness. The precuneus, PCC, and retrosplenial cortex are closely related to each other, forming a resting network of the human brain that spontaneously and selectively connects with other parietal lobe regions. That is, the visual-spatial network includes the caudate nucleus, superior and inferior parietal lobules, and intraparietal sulcus. The central cortex of the precuneus is also related to the frontal lobe, and it participates in facial recognition together with the "fusiform face area" [39]. In previous studies on diseases related to visual dysfunction, an abnormal FCD of the precuneus was also found. For example, the FCD of the precuneus was increased in patients with multiple sclerosis [40]. However, studies on glaucoma found that the FCD between the precuneus and the relevant cortexes was decreased $[41,42]$. In this study, we found that the FCD of the left precuneus was increased in the patients with DON, the relevant brain areas included the left middle temporal gyrus, the right cuneus, the right fusiform gyrus, and the left superior occipital gyrus. These areas are closely related to the dorsal visual pathway and facial recognition. The increase in FCD values may be related to an increase in compensatory connectivity after visual pathway damage.

The dorsal pathway of the visual cognitive pathway depends on the P cells of the optic ganglion cells. Therefore, the dorsal pathway's critical point is closely related to the peripheral VFs [43]. The hippocampus is a part of the temporal lobe, so selective surgical excision of the amygdala or temporal lobe would result in homonymous quadrantanopia [44]. This study revealed that the MD of the VF in DON patients was correlated with the FCD of the central cortex, indicating that DON patients' central cor-

A New Pathogenesis Hypothesis of DON tical injury may participate in the change in the VF. Besides, there was no significant difference in the thickness of the optic nerve fiber layer between the DON patients and the nDON patients, and there was no correlation between the thickness of the optic nerve fiber layer and the VF MD, which also supported the above view to some extent.

Information processing in the brain shows laterality, such as language and auditory sensation. Visual processing also showed laterality [45-47]. The symptoms of neglect in the right hemisphere damage were more frequent and severe than those in the left hemisphere, which was considered a sign of lateralization of the right hemisphere in the process of visual attention [48-50]. Falasca NW et al. found that top-down visual information processing was only related to the right frontal gyrus [45]. In this study, only the right frontal lobe was abnormal, which may be related to this laterality.

Both hypothyroidism and hyperthyroidism can lead to abnormalities in brain structures and functions [20, 24, $51,52]$. A study, including 2,557 patients, showed that patients with severe hypothyroidism experienced a decrease in total brain volume [49]. The resting-state MRI study showed the brain functional connectivity of the right anterior parietal lobe network, medial visual network, and the motor network was significantly reduced in patients with hypothyroidism [21]. Compared with the control group, the F.C. of the bilateral anterior insular lobe, bilateral posterior insular lobe, and left anterior cerebellar lobe were higher in patients with hyperthyroidism. In contrast, the F.C. of the bilateral lateral prefrontal cortices, medial temporal lobe, and bilateral PCC were lower [53]. Even short-course hyperthyroidism could cause abnormal brain connectivity [54].

Although there are no studies on the effect of dynamic changes in thyroid function on the structures of brain regions, Krausz et al. [55] compared regional cerebral blood flow between patients with hypothyroidism and healthy subjects. They then evaluated the cerebral blood flow of these patients when they regained normal thyroid function after treatment. Compared with the control group, patients with hypothyroidism had lower regional cerebral blood flow in the right paraoccipital gyrus, cuneus, PCC, lingual gyrus, fusiform gyrus, insula, and preand postcentral gyri before the treatment. After the treatment, the perfusion of these patients did not return to normal while the thyroid function returned to normal. These findings were confirmed by later studies [56-58].

As mentioned above, dynamic abnormality in the thyroid hormone levels can lead to the visual cortex's struc- 
tural and functional abnormalities. Previous studies have shown significant fluctuation of thyroid function in the part of TAO patients during the treatment process [50]. Therefore, combined with this study's results, we speculated that the dynamic abnormality of thyroid might be involved in the abnormality of FCD in DON patients. Although there is no significant correlation between the thyroid hormone level and FCD in this study, one possible reason is that the thyroid function in this study is determined by a single detection level, which cannot reflect the dynamic changes of its impact on FCD, and thus, further research is needed.

In summary, the abnormal FCD in the cortex of DON patients suggests that a central nervous system mechanism may be related to the pathogenesis of the DON. The dynamic abnormality of thyroid might be involved in the abnormality of FCD in DON patients, which should be further researched. The disadvantages of this study are the lack of follow-up results concerning dynamic thyroid hormone abnormalities, and the small sample size brain function, which needs to be further expanded and analyzed.

\section{Acknowledgements}

The authors thank Dr.Tingting Chen and Dr. Bnngxun Mao for collecting the clinical data.

\section{Statement of Ethics}

Ethical approval was approved by the Ethics Committee Board of theEyeHospital of Wenzhou MedicalUniversity(KYK[2013]27). The subjects had given written informed consent and all the procedures being performed were part of the routine care.

\section{Conflict of Interest Statement}

The authors have no conflicts of interest to declare.

\section{Funding Sources}

This study was financially supported by the Zhejiang provincial Natural Science Foundation of China under Grant No. LY19H120003 and the Eye Hospital of Wenzhou Medical University (YNCX201314) and Medical Health Science and Technology Project of Zhejiang Provincial Health Commission (Grant No. 2020KY192).

\section{Author Contributions}

Y.H.T. and J.L.G. conceived and designed the study. P.P.H. and C.W.M. performed the experiments. Y.H.T. wrote the paper. Y.H.T., X.Z.L., and J.L.G. reviewed and edited the manuscript. All authors read and approved the manuscript.

\section{References}

1 Blandford AD, Zhang D, Chundury RV, Perry JD. Dysthyroid optic neuropathy: update on pathogenesis, diagnosis, and management. Expert Rev Ophthalmol. 2017;12(2):111-21.

2 Kazim M, Trokel SL, Acaroglu G, Elliott A. Reversal of dysthyroid optic neuropathy following orbital fat decompression. Br J Ophthalmol. 2000;84(6):600-5.

3 Lima Bda R, Perry JD. Superior ophthalmic vein enlargement and increased muscle index in dysthyroid optic neuropathy. Ophthalmic Plast Reconstr Surg. 2013;29(3):147-9.

4 Gonçalves AC, Silva LN, Gebrim EM, Monteiro ML. Quantification of orbital apex crowding for screening of dysthyroid optic neuropathy using multidetector C.T. AJNR Am J Neuroradiol. 2012;33(8):1602-7.

5 Weis E, Heran MK, Jhamb A, Chan AK, Chiu JP, Hurley MC, et al. Quantitative computed tomographic predictors of compressive optic neuropathy in patients with thyroid orbitopathy: a volumetric analysis. Ophthalmology. 2012;119(10):2174-8.

6 Tong Y, Chen G, Jiang F, Wu W. Successful delayed treatment of the traumatic orbital apex syndrome by nasal endoscopic decompression surgery. Indian J Ophthalmol. 2015; 63(9):728-30.
7 Jeon C, Shin JH, Woo KI, Kim YD. Clinical profile and visual outcomes after treatment in patients with dysthyroid optic neuropathy. Korean J Ophthalmol. 2012;26(2):73-9.

8 Lin HC, Kang JH, Jiang YD, Ho JD. Hypothyroidism and the risk of developing open-angle glaucoma: a five-year population-based followup study. Ophthalmology. 2010;117(10):1960-6.

9 Gopinath B, Liew G, Kifley A, Mitchell P. Thyroid dysfunction and ten-year incidence of age-related macular degeneration. Invest Ophthalmol Vis Sci. 2016;57(13):5273-7.

10 Chaker L, Buitendijk GH, Dehghan A, Medici M, Hofman A, Vingerling JR, et al. Thyroid function and age-related macular degeneration: a prospective population-based cohort studythe Rotterdam Study. BMC Med. 2015;13:94.

11 Cakir M, Turgut Ozturk B, Turan E, Gonulalan G, Polat I, Gunduz K. The effect of hypothyroidism on color contrast sensitivity: a prospective study. Eur Thyroid J. 2015;4(1):43-7.

12 Jaiswal P, Saxena Y, Gupta R, Kaushik RM. Pattern reversal visual evoked potential and cognitive functions in subclinical hypothyroid subjects. J Neurosci Rural Pract. 2016; 7(Suppl 1):S46-51.

13 Tamburini G, Tacconi P, Ferrigno P, Cannas A, Massa GM, Mastinu R, et al. Visual evoked potentials in hypothyroidism: a long-term evaluation. Electromyogr Clin Neurophysiol. 1998;38(4):201-5.

14 Yamamoto K, Saito K, Takai T, Naito M, Yoshida S. Visual field defects and pituitary enlargement in primary hypothyroidism. J Clin Endocrinol Metab. 1983;57(2):283-7.

15 Glaschke A, Weiland J, Del Turco D, Steiner M, Peichl L, Glösmann M. Thyroid hormone controls cone opsin expression in the retina of adult rodents. J Neurosci. 2011;31(13):4844-51.

16 Remaud S, Ortiz FC, Perret-Jeanneret M, Aigrot MS, Gothié JD, Fekete C, et al. Transient hypothyroidism favors oligodendrocyte generation providing functional remyelination in the adult mouse brain. Elife. 2017;6: e29996.

17 Fernandez M, Pirondi S, Manservigi M, Giardino L, Calzà L. Thyroid hormone participates in the regulation of neural stem cells and oligodendrocyte precursor cells in the central nervous system of adult rat. Eur J Neurosci. 2004;20(8):2059-70.

18 Tokumoto Y, Tamaki S, Kabe Y, Takubo K, Suematsu M. Quiescence of adult oligodendrocyte precursor cells requires thyroid hormone and hypoxia to activate Runx1. Sci Rep. 2017;7(1):1019. 
19 Correia N, Mullally S, Cooke G, Tun TK, Phelan N, Feeney J, et al. Evidence for a specific defect in hippocampal memory in overt and subclinical hypothyroidism. J Clin Endocrinol Metab. 2009;94(10):3789-97.

20 Göbel A, Heldmann M, Göttlich M, Dirk AL, Brabant G, Münte TF. Effect of experimental thyrotoxicosis on brain gray matter: a voxelbased morphometry study. Eur Thyroid J. 2015;4(Suppl 1):113-8.

21 Singh S, Kumar M, Modi S, Kaur P, Shankar LR, Khushu S. Alterations of functional connectivity among resting-state networks in hypothyroidism. J Neuroendocrinol. 2015; 27(7):609-15.

22 Hasegawa M, Kida I, Wada H. A volumetric analysis of the brain and hippocampus of rats rendered perinatal hypothyroid. Neurosci Lett. 2010;479(3):240-4.

23 YinLiao JJLM, Luo DX, Xu K, Ma SH, Wang ZX, Le HB, et al. Spatial working memory impairment in subclinical hypothyroidism: an FMRI study. Neuroendocrinology. 2013; 97(3):260-70.

24 Singh S, Modi S, Bagga D, Kaur P, Shankar LR, Khushu S. Voxel-based morphometric analysis in hypothyroidism using diffeomorphic anatomic registration via an exponentiated lie algebra algorithm approach. J Neuroendocrinol. 2013;25(3):229-34.

25 Hendelman WJ, Inc T. Atlas de neuroanatomie fonctionnelle: première édition française. 2013.

26 Singh S, Trivedi R, Singh K, Kumar P, Shankar LR, Khushu S. Diffusion tensor tractography in hypothyroidism and its correlation with memory function. J Neuroendocrinol. 2014;26(11):825-33.

27 Shin YW, Choi YM, Kim HS, Kim DJ, Jo HJ, O'Donnell BF, et al. Diminished quality of life and increased brain functional connectivity in patients with hypothyroidism after total thyroidectomy. Thyroid. 2016;26(5):641-9.

28 Tomasi D, Volkow ND. Functional connectivity density mapping. Proc Natl Acad Sci USA. 2010;107(21):9885-90.

29 Bartley GB, Gorman CA. Diagnostic criteria for Graves' ophthalmopathy. Am J Ophthalmol. 1995;119(6):792-5

$30 \mathrm{Hu}$ Y, Liu X, Chen X, Chen T, Ye P, Jiang L, et al. Differences in the functional connectivity density of the brain between individuals with growth hormone deficiency and idiopathic short stature. Psychoneuroendocrinology. 2019;103:67-75.

31 Guo Z, Liu X, Hou H, Wei F, Liu J, Chen X. Abnormal degree centrality in Alzheimer's disease patients with depression: a restingstate functional magnetic resonance imaging study. Exp Gerontol. 2016;79:61-6.

32 Kravitz DJ, Saleem KS, Baker CI, Mishkin M. A new neural framework for visuospatial processing. Nat Rev Neurosci. 2011;12(4):217-30.
33 Creem SH, Proffitt DR. Defining the cortical visual systems: "what", "where", and "how". Acta Psychol. 2001;107(1-3):43-68.

34 Galletti C, Fattori P. The dorsal visual stream revisited: stable circuits or dynamic pathways. Cortex. 2018;98:203-17.

35 Wang Z, Yue L, Cui C, Liu S, Wang X, Li Y, et al. Top-down control of the medial orbitofrontal cortex to nucleus accumbens core pathway in decisional impulsivity. Brain Struct Funct. 2019;224(7):2437-52.

36 Dijkstra N, Bosch SE, van Gerven MAJ. Shared neural mechanisms of visual perception and imagery. Trends Cogn Sci. 2019; 23(5):423-34.

37 Hu F, Kamigaki T, Zhang Z, Zhang S, Dan U, Dan Y. Prefrontal corticotectal neurons enhance visual processing through the superior colliculus and pulvinar thalamus. Neuron. 2019;104(6):1141-e4.

38 Bar M, Kassam KS, Ghuman AS, Boshyan J, Schmid AM, Schmidt AM, et al. Top-down facilitation of visual recognition. Proc Natl Acad Sci USA. 2006;103(2):449-54.

39 Cox D, Meyers E, Sinha P. Contextually evoked object-specific responses in human visual cortex. Science. 2004;304(5667):115-7.

40 Petsas N, De Giglio L, González-Quintanilla V, Giuliani M, De Angelis F, Tona F, et al. Functional connectivity changes after initial treatment with fingolimod in multiple sclerosis. Front Neurol. 2019;10:153.

41 Wu YY, Wang SF, Zhu PW, Yuan Q, Shi WQ Lin $\mathrm{Q}$, et al. Altered intrinsic functional connectivity of the primary visual cortex in patients with neovascular glaucoma: a resting-state functional magnetic resonance imaging study. Neuropsychiatr Dis Treat. 2020;16:25-33.

42 Wang B, Yan T, Zhou J, Xie Y, Qiu J, Yi W, et al. Altered fMRI-derived functional connectivity in patients with high-tension glaucoma. J Neuroradiol. 2020;S0150-9861:30126-7.

43 Blatt GJ, Andersen RA, Stoner GR. Visual receptive field organization and cortico-cortical connections of the lateral intraparietal area (area LIP) in the macaque. J Comp Neurol. 1990;299(4):421-45

44 Mengesha T, Abu-Ata M, Haas KF, Lavin PJ, Sun DA, Konrad PE, et al. Visual field defects after selective amygdalohippocampectomy and standard temporal lobectomy. J Neuroophthalmol. 2009;29(3):208-13.

45 Falasca NW, D'Ascenzo S, Di Domenico A Onofrj M, Tommasi L, Laeng B, et al. Hemispheric lateralization in top-down attention during spatial relation processing: a Granger causal model approach. Eur J Neurosci. 2015; 41(7):914-24.

46 Franklin A, Catherwood D, Alvarez J, Axelsson E. Hemispheric asymmetries in categorical perception of orientation in infants and adults. Neuropsychologia. 2010;48(9):2648-57.
47 Siman-Tov T, Mendelsohn A, Schonberg T, Avidan G, Podlipsky I, Pessoa L, et al. Bihemispheric leftward bias in a visuospatial attention-related network. J Neurosci. 2007;27(42): 11271-8.

48 Benwell CS, Thut G, Grant A, Harvey M. A rightward shift in the visuospatial attention vector with healthy aging. Front Aging Neurosci. 2014;6:113.

49 Stam CJ, Bakker M. The prevalence of neglect: superiority of neuropsychological over clinical methods of estimation. Clin Neurol Neurosurg. 1990;92(3):229-35.

50 Hatin B, Sykes Tottenham L, Oriet C. The relationship between collisions and pseudoneglect: is it right. Cortex. 2012;48(8):997-1008.

51 Ittermann T, Wittfeld K, Nauck M, Bülow R, Hosten N, Völzke H, et al. High thyrotropin is associated with reduced hippocampal volume in a population-based study from Germany. Thyroid. 2018;28(11):1434-42.

52 Zhang W, Song L, Yin X, Zhang J, Liu C, Wang J, et al. Grey matter abnormalities in untreated hyperthyroidism: a voxel-based morphometry study using the DARTEL approach. Eur J Radiol. 2014;83(1):e43-8.

53 Liu B, Ran Q, Liu D, Zhang S, Zhang D. Changes in resting-state cerebral activity in patients with hyperthyroidism: a short-term follow-up functional MR imaging study. Sci Rep. 2017;7(1):10627.

54 Göttlich M, Heldmann M, Göbel A, Dirk AL, Brabant G, Münte TF. Experimentally induced thyrotoxicosis leads to increased connectivity in temporal lobe structures: a resting state fMRI study. Psychoneuroendocrinology. 2015;56:100-9.

55 Krausz Y, Freedman N, Lester H, Newman JP, Barkai G, Bocher M, et al. Regional cerebral blood flow in patients with mild hypothyroidism. J Nucl Med. 2004;45(10):1712-5.

56 Nagamachi S, Jinnouchi S, Nishii R, Ishida Y, Fujita S, Futami S, et al. Cerebral blood flow abnormalities induced by transient hypothyroidism after thyroidectomy-analysis by tc-99m-HMPAO and SPM96. Ann Nucl Med. 2004;18(6):469-77.

57 Kaichi Y, Kenjo M, Higaki T, Tatsugami F, Akiyama Y, Nagata Y, et al. Cerebral blood flow in transient hypothyroidism after thyroidectomy: arterial spin labeling magnetic resonance study. Neuro Endocrinol Lett. 2015;36(6):545-51.

58 Kaya M, Cermik TF, Bedel D, Kutucu Y, Tuglu C, Yigitbasi ON. Assessment of alterations in regional cerebral blood flow in patients with hypothyroidism due to Hashimoto's thyroiditis. J Endocrinol Invest. 2007;30(6): 491-6. 